\title{
Algebraic Solution to Minimum-Time Velocity Planning
}

\author{
Gabriele Lini, Aurelio Piazzi*, and Luca Consolini
}

\begin{abstract}
The paper poses the problem of minimum-time velocity planning subject to a jerk amplitude constraint and to arbitrary velocity/acceleration boundary conditions. This problem which is relevant in the field of autonomous robotic navigation and also for inertial one-dimensional mechatronics systems is dealt with an algebraic approach based on Pontryagin's Maximum Principle. The exposed complete solution shows how this time-optimal planning can be reduced to the problem of determining the positive real roots of a quartic equation. An algorithm that is suitable for real-time applications is then presented. The paper includes detailed examples also highlighting the special cases of this planning problem.
\end{abstract}

Keywords: Autonomous robotic navigation, feedforward control, iterative steering, minimum-time control, Pontryagin's Maximum Principle, velocity planning.

\section{INTRODUCTION}

In the field of robotics, much research has focused on minimum-time problems because solving them helps to achieve high-performance or to maximize production. For instance, an effective algorithm to move a robotic manipulator in minimum-time along a specified geometric path subject to input torque/force constraints was presented by Shin and McKay in [1]. In another instance, taken from autonomous robotic navigation, a unicycle mobile robot subject to linear and angular velocity bounds can be moved in minimum-time from a given configuration to another one by using ReedsShepps curves [2,3].

Still in the field of autonomous robotic navigation, the work presented in this paper faces the minimum-time (longitudinal) velocity planning in the context of the socalled path-velocity decomposition [4] and the iterative steering technique [5-7]. The robot vehicle has to travel on an assigned geometric path of a given length and the longitudinal vehicle velocity can be planned by seeking a time-optimal (i.e., minimum-time) motion subject to a jerk amplitude constraint and to arbitrary velocity/ acceleration boundary conditions. The continuousacceleration profile with a given bound on the jerk (i.e.,

Manuscript received July 1, 2011; revised July 25, 2012 and March 8, 2013; accepted March 11, 2013. Recommended by Editorial Board member Duk-Sun Shim under the direction of Editor Hyouk Ryeol Choi.

This paper is a revised and expanded version of a work presented at the Seventh International Workshop on Robot Motion and Control, Czerniejewo (Poland), 1-3 June 2009 [17].

Gabriele Lini is with SISSA, International School for Advanced Studies, Via Bonomea 265, I-34136, Trieste, Italy (e-mail: gabriele.lini@sissa.it).

Aurelio Piazzi and Luca Consolini are with the Department of Information Engineering, University of Parma, Parco Area delle Scienze 181A, I-43124, Parma, Italy (e-mails: \{aurelio.piazzi, luca.consolini\}@unipr.it).

* Corresponding author. the time derivative of the acceleration) facilitates the implementation of the time-optimal movement and the interpolation of arbitrarily given velocity and acceleration at the endpoints of the planned time interval permits the supervisor of the iterative steering strategy to perform real-time sensor-based navigation while ensuring an overall smoothness of the vehicle motion [5].

The importance of minimum-time velocity planning with arbitrary boundary conditions was first pointed out by Koh et al. [8] in 1999 in the context of robotic and mechatronics systems with real-time motion planning applications. In subsequent years, velocity planning for autonomous navigation was dealt with cubic (polynomial) splines schemes and parametric local optimization to achieve minimum-time optimality in static and dynamic environments [9] or minimum-jerk optimality when a prefixed time interval is given [10-12]. These approaches use fast and efficient optimization algorithms and can deal with velocity and acceleration constraints, but they all cannot achieve true optimality because the function space where to search the optimizer is restricted by the choice of the polynomial splines scheme and moreover, the optimization algorithms can only converge on local minima. An application of [10] to the velocity planning for autonomous passenger vehicles was presented in [13] to achieve travel comfort with low values of acceleration and jerk. For an automated assembly manufacturing, a fifth order splines scheme was adopted in [14] to obtain a velocity planning that minimizes the time integral of the squared jerk. A comprehensive reference on velocity planning for automatic machines and robots can be found in [15]; more general trajectory planning methods and algorithms are presented in [16].

The addressed minimum-time velocity planning problem will be easily recast into an input-constrained reachability control problem for the triple integrator system (cf. (6)), where the time-optimal control input is actually the second time-derivative (jerk) of the sought 
velocity profile. Hence, by virtue of the Pontryagin's Maximum Principle, the time-optimal control is a bangbang function with at most two switchings. The paper presents an algebraic approach to obtain this time-optimal control jerk (and then the minimum-time velocity profile) and an algorithm to compute it. The exposed algebraic solution shows that the addressed problem can be reduced to the problem of determining the positive real roots of a quartic equation whose coefficients depend on the problem data, i.e., the path length, the jerk bound, and the velocities and accelerations at the endpoints of the planned time interval. This implies that the addressed minimum-time planning is solvable in a few steps, and consequently real-time applications of the devised algorithm are practicable.

The paper is organized as follows. Section 2 states the minimum-time velocity planning problem and presents the structure of the corresponding time-optimal jerk. The case of initial or final nil conditions (i.e., when velocity and acceleration are zero at the initial or final planning time) is also addressed and preliminary results are exposed (Proposition 1 and Corollary 1). The next Section 3 presents the algebraic solution of the planning problem. The solution set $S_{\mathbf{p}}$ associated to the nonlinear algebraic systems (17) is introduced and the reduction of this system to the single quartic equation (23) is exposed. Two subsections treat the special planning cases: subsection 3.1 considers the degenerate case (cf. Proposition 3) and subsection 3.2 provides a result on the cardinality of $S_{\mathbf{p}}$ (cf. Proposition 4) for the case of initial or final nil conditions. The algorithm to compute the time-optimal jerk solution is reported in Section 4. Examples are illustrated in Section 5 and final remarks end the paper in Section 6. The presented exposition is a revised and expanded version of the conference paper [17]. Some of the improvements over [17] are: (i) introduction of the case of initial or final nil conditions; (ii) reduction to a quartic equation instead to a fifth one; (iii) introduction of the (algebraic) solution set $S_{\mathbf{p}}$; (iv) rigorous analysis of the degenerate case.

Notation (piecewise $C^{2}$-continuity): A function $f: \mathbb{R} \rightarrow \mathbb{R}, t \rightarrow f(t)$ has $P C^{2}$ continuity, and we say $f(t) \in P C^{2}$ if (1) $f(t) \in C^{1}(\mathbb{R}) ; \quad(2) \quad f(t) \in C^{2}(\mathbb{R}-$ $\left.\left\{t_{1}, t_{2}, \ldots\right\}\right)$; (3) $\exists \lim _{t \rightarrow t_{j}^{-}} D^{2} f(t), \quad \exists \lim _{t \rightarrow t_{i}^{+}} D^{2} f(t)$, $i=1,2, \ldots$ where $\left\{t_{1}, t_{2}, \ldots\right\}$ is a set of discontinuity instants.

\section{PROBLEM STATEMENT AND THE STRUCTURE OF THE OPTIMAL SOLUTION}

The problem solved in this paper is the planning of a minimum-time smooth velocity profile $v(t) \in P C^{2}$ where the jerk does not exceed a given bound and the initial and final conditions on the velocity and acceleration are arbitrarily assigned.

Formally, the Minimum-Time Velocity Planning (MTVP) problem can be stated as follows:

$$
\min _{v \in P C^{2}} t_{f}
$$

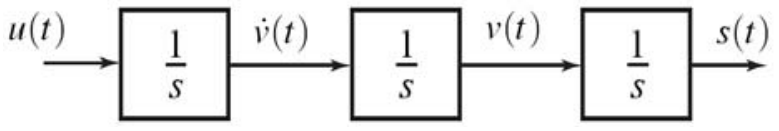

Fig. 1. The triple integrator system for velocity planning.

such that

$$
\begin{aligned}
& \int_{0}^{t_{f}} v(\xi) d \xi=s_{f}, \\
& v(0)=v_{0}, \quad v\left(t_{f}\right)=v_{f}, \\
& \dot{v}(0)=a_{0}, \quad \dot{v}\left(t_{f}\right)=a_{f}, \\
& |\ddot{v}(t)| \leq j_{M} \quad \forall t \in\left[0, t_{f}\right],
\end{aligned}
$$

where $t_{f}$ is the traveling time to complete a path whose length is $s_{f}>0$ (or also $s_{f}<0$ in case a backward movement is prescribed), the maximum allowed jerk value is $j_{M}>0$ and $v_{0}, v_{f} \in \mathbb{R}, a_{0}, a_{f} \in \mathbb{R}$ are arbitrary velocities and accelerations imposed at the endpoints of interval $\left[0, t_{f}\right]$ (they form the boundary conditions of the problem). The solution of the above problem is denoted by $v^{*}(t) \in P C^{2}$ with associated minimum-time $t_{f}^{*}$.

The MTVP problem can be easily recast to an inputconstrained minimum-time control problem with respect to the triple integrator system. Indeed consider the jerk $\ddot{v}(t)$ as the control input $u(t)$ of a cascade of three integrators as depicted in Fig. 1.

Introduce the state $\mathbf{x}(t)$ as the column vector

$$
\left[\begin{array}{l}
x_{1}(t) \\
x_{2}(t) \\
x_{3}(t)
\end{array}\right]:=\left[\begin{array}{c}
s(t) \\
v(t) \\
\dot{v}(t)
\end{array}\right]
$$

and the triple integrator model is as follows

$$
\dot{\mathbf{x}}(t)=\left[\begin{array}{lll}
0 & 1 & 0 \\
0 & 0 & 1 \\
0 & 0 & 0
\end{array}\right] \mathbf{x}(t)+\left[\begin{array}{l}
0 \\
0 \\
1
\end{array}\right] u(t)
$$

Hence, the problem (1)-(5) is equivalent to find the timeoptimal control $u^{*}(t)$ that brings the state of system (6) from the initial state $\mathbf{x}_{0}=\left[\begin{array}{lll}0 & v_{0} & a_{0}\end{array}\right]^{T}$ to the final state $\mathbf{x}_{f}=\left[\begin{array}{lll}s_{f} & v_{f} & a_{f}\end{array}\right]^{T}$ in minimum time $t_{f}^{*}$, while satisfying the input constraint

$$
\left|u^{*}(t)\right| \leq j_{M} \quad \forall t \in\left[0, t_{f}^{*}\right] .
$$

In this context, from the Pontryagin's Maximum Principle it is known that there exists a unique timeoptimal $u^{*}(t)$ which is a classic bang-bang control function, i.e., a function that switches between the minimum and maximum allowed values (in our problem $-j_{M}$ and $\left.+j_{M}\right)$. A relevant result of the Pontryagin's Maximum Principle is the following.

Theorem 1 [18]: Let be given a controllable $n$-th order system $\dot{\mathbf{x}}=\mathbf{A x}+\mathbf{b} u$ with any given amplitude 
input constraint $c>0$. Consider the unique timeoptimal bang-bang control $u^{*}(t)$ that brings the system from a given initial state $\mathbf{x}(0)=\mathbf{x}_{0} \in \mathbb{R}^{n}$ to a given final state $\mathbf{x}\left(t_{f}^{*}\right)=\mathbf{x}_{f} \in \mathbb{R}^{n}$ in minimum-time $t_{f}^{*}$ while satisfying $\left|u^{*}(t)\right| \leq c \forall t \in\left[0, t_{f}^{*}\right]$. If all the eigenvalues of the system matrix A are real the time-optimal $u^{*}(t)$ has at most $n-1$ switching times.

The following proposition, which derives from a Sontag's result [19], is also relevant.

Proposition 1: Let be given a controllable $n$-th order system $\dot{\mathbf{x}}=\mathbf{A x}+\mathbf{b} u$ with any given amplitude input constraint $c>0$ and assume that all the eigenvalues of A are real. Consider initial and final states $\mathbf{x}_{0}, \mathbf{x}_{f} \in \mathbb{R}^{n}$ for which $\mathbf{x}_{0}=0$ or $\mathbf{x}_{f}=0$. In both cases if a bangbang control $u(t)$ (i.e., an input function that switches between the constant values $-c$ and $+c$ ) with at most $n-1$ switching times steers the state from $\mathbf{x}_{0}$ to $\mathbf{x}_{f}$ then $u(t)$ is the unique time-optimal control that steers $\mathbf{x}_{0}$ to $\mathbf{x}_{f}$ in minimum-time.

Proof: Since the system is controllable and either the initial or final state is 0, Proposition 10.2.9 in [19] guarantees that if $u$ satisfies the maximum principle, then it is time-optimal. It remains to show that control $u(t)$, $t \in\left[0, t_{f}\right]$ ( $t_{f}$ is the associated steering time) satisfies indeed the maximum principle, i.e., there exists a vector $\eta \in \mathbb{R}^{n}$ (the initial costate) such that

$$
u(t)=\operatorname{sign}\left(\eta^{T} e^{-\mathbf{A} t} \mathbf{b}\right) c \text { for almost all } t \in\left[0, t_{f}\right],
$$

where "sign" is the standard sign function. To this end, let $t_{1}, t_{2}, \ldots, t_{k} \in\left(0, t_{f}\right)$ be the switching times of $u$, with $k<n$. In case $k<n-1$, choose additional distinct times $t_{k+1}, t_{k+2}, \ldots, t_{n-1}>t_{f}$ arbitrarily. It can be always assumed $u(0) \neq 0$. Define the initial costate $\eta \in \mathbb{R}^{n}$ as the unique solution of the linear system

$$
\left\{\begin{array}{l}
\mathbf{b}^{T} \eta=\operatorname{sign}(u(0)) \\
\mathbf{b}^{T} e^{-\mathbf{A}^{T} t_{i}} \eta=0, \quad i=1, \ldots, n-1 .
\end{array}\right.
$$

System (9) has indeed a solution which is unique because matrix

$$
\mathbf{M}=\left[\mathbf{b}, e^{-\mathbf{A} t_{1}} \mathbf{b}, \ldots, e^{-\mathbf{A} t_{n-1}} \mathbf{b}\right]
$$

is nonsingular. In fact, assume by contradiction that $\mathbf{M}$ is singular, then there exist $\lambda \in \mathbb{R}^{n}$ such that $\lambda^{T} \mathbf{M}=0$, this implies that function $g(t)=\lambda^{T} e^{-\mathbf{A} t} \mathbf{b}$ satisfies

$$
g(t)=0, \quad t=0, t_{1}, \ldots, t_{n-1} .
$$

Function $g$ is a quasipolynomial that has $n$ distinct real roots. Hence, it must be identically zero, i.e., $g(t) \equiv 0$, because any quasipolynomial that is not identically zero cannot have more than $n-1$ distinct real roots (see [20], Lemma 15.1). Therefore, $g$ and its derivatives are all identically zero and by explicit evaluation at $t=0$ it follows that

$$
\lambda^{T}\left[\mathbf{b}, \mathbf{A b}, \ldots, \mathbf{A}^{n-1} \mathbf{b}\right]=0,
$$

which means that pair $(\mathbf{A}, \mathbf{b})$ is not controllable and this contradicts the hypothesis. Hence, there exists $\eta \neq 0$ satisfying relations (9) for which function $\eta^{T} e^{-\mathbf{A} t} \mathbf{b}$ is a quasi-polynomial that has globally $n-1$ distinct roots and over the open interval $\left(0, t_{f}\right)$ it has exactly the $k$ roots $t_{1}, t_{2}, \ldots, t_{k}$ and no other real root. Since

$$
\operatorname{sign}\left(\left.\eta^{T} e^{-\mathbf{A} t} \mathbf{b}\right|_{t=0}\right)=\operatorname{sign}(u(0))
$$

it then follows that control $u$ satisfies statement (8) and this completes the proof.

Considering that system (6) is the triple integrator model that has three null eigenvalues, from Theorem 1 it follows that the time-optimal jerk $u^{*}(t)$ has at most two switching instants. Hence, the structure of the optimal $u^{*}(t)$ is depicted in Fig. 2 where $u_{M} \in\left\{-j_{M},+j_{M}\right\}$ and $0 \leq t_{1} \leq t_{2} \leq t_{3}=t_{f}^{*}$ with $t_{f}^{*}>0$. Once the time-optimal jerk could been found, the time-optimal velocity would be straightforwardly given by

$$
v^{*}(t)=v_{0}+a_{0} t+\int_{0}^{t}\left(\int_{0}^{\eta} u^{*}(\xi) d \xi\right) d \eta, t \in\left[0, t_{f}^{*}\right] .
$$

Corollary 1: Consider the MTVP problem with initial nil conditions, i.e., $v_{0}=0, a_{0}=0$, or final nil conditions, i.e., $v_{f}=0, a_{f}=0$. If a bang-bang control $u(t)$ with at most 2 switching times steers the state of system (6) from the initial state $\mathbf{x}_{0}=\left[\begin{array}{lll}0 & v_{0} & a_{0}\end{array}\right]^{T}$ to the final state $\mathbf{x}_{f}=\left[s_{f}\right.$ $\left.v_{f} a_{f}\right]^{T}$ then $u(t)$ is the unique time-optimal control that steers $\mathbf{x}_{0}$ to $\mathbf{x}_{\mathbf{f}}$ in minimum-time.

Proof: If $v_{0}=0, a_{0}=0$ then $\mathbf{x}_{0}=0$ so that the thesis of the corollary is an immediate consequence of Proposition 1. When $v_{f}=0, a_{f}=0$ we note that a control $u(t)$ steers system (6) from $\left[\begin{array}{lll}0 & v_{0} & a_{0}\end{array}\right]^{T}$ to $\left[\begin{array}{lll}s_{f} & 0 & 0\end{array}\right]^{T}$ if and only if $u(t)$ steers system (6) from $\left[\begin{array}{lll}-s_{f} & v_{0} & a_{0}\end{array}\right]^{T}$ to $\left[\begin{array}{lll}0 & 0 & 0\end{array}\right]^{T}$. Hence, application of Proposition 1 in the case $\mathbf{x}_{f}=0$ completes the proof.

\section{THE ALGEBRAIC SOLUTION}

The structure of the time-optimal control jerk $u^{*}(t)$ associated to the MTVP problem (1)-(5) - as exposed in the previous section leads to the algebraic approach to determine it. Indeed, the problem is reduced to finding, by exploitation of the boundary conditions (2)-(4), the switching time values $t_{1}$ and $t_{2}$, the prospective minimum-time $t_{3}$, and the sign of the jerk initial value $u_{M}$ (corresponding to the positive $+j_{M}$ or the negative $-j_{M}$ ). These switching times must satisfy the ordering $0 \leq t_{1} \leq t_{2} \leq t_{3}$ with $t_{3}>0$.

From (6) and the initial conditions, the acceleration profile $x_{3}(t)$ can be computed according to

$$
x_{3}(t)=a_{0}+\int_{0}^{t} u(\xi) d \xi, \quad t \in\left[0, t_{3}\right]
$$

where $u(t)$ is the prospective optimal jerk to be defined as follows (cf. Fig. 2): 


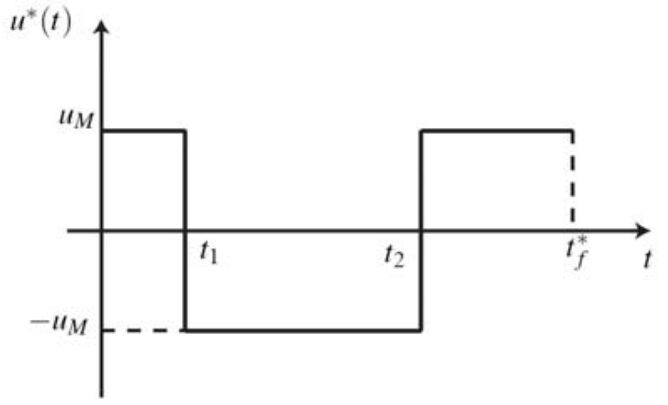

Fig. 2. The minimum-time control (jerk) profile.

$$
u(t)=\left\{\begin{array}{lll}
u_{M} & \text { if } & t \in\left[0, t_{1}\right] \\
-u_{M} & \text { if } & t \in\left(t_{1}, t_{2}\right] \\
u_{M} & \text { if } & t \in\left(t_{2}, t_{3}\right] .
\end{array}\right.
$$

By integration of the jerk profile, the corresponding acceleration is:

$$
x_{3}(t)= \begin{cases}a_{0}+u_{M} t & t \in\left[0, t_{1}\right] \\ a_{0}+2 u_{M} t_{1}-u_{M} t & t \in\left[t_{1}, t_{2}\right] \\ a_{0}+2 u_{M} t_{1}-2 u_{M} t_{2}+u_{M} t & t \in\left[t_{2}, t_{3}\right] .\end{cases}
$$

From interpolation of the acceleration at the final time $t_{3}$, i.e., $x_{3}\left(t_{3}\right)=a_{f}$, it emerges an equation which is linear in the unknown $t_{1}, t_{2}$, and $t_{3}$ :

$$
2 u_{M} t_{1}-2 u_{M} t_{2}+u_{M} t_{3}=a_{f}-a_{0} \text {. }
$$

Considering the initial condition on velocity and integrating the second equation of state model (6), the velocity profile $x_{2}(t)$ arises as follows:

$$
\begin{aligned}
& x_{2}(t)= \\
& \begin{cases}v_{0}+a_{0} t+\frac{1}{2} u_{M} t^{2} & t \in\left[0, t_{1}\right] \\
v_{0}-u_{M} t_{1}^{2}+a_{0} t+2 u_{M} t_{1} t-\frac{1}{2} u_{M} t^{2} & t \in\left[t_{1}, t_{2}\right] \\
v_{0}-u_{M} t_{1}^{2}+u_{M} t_{2}^{2}+a_{0} t+2 u_{M} t_{1} t & \\
-2 u_{M} t_{2} t+\frac{1}{2} u_{M} t^{2} & t \in\left[t_{2}, t_{3}\right] .\end{cases}
\end{aligned}
$$

Interpolating the final velocity with relation $x_{2}\left(t_{3}\right)=v_{f}$ we obtain an equation which is quadratic in $t_{1}, t_{2}$, and $t_{3}$ :

$$
\begin{aligned}
-u_{M} t_{1}^{2} & +2 u_{M} t_{1} t_{3}+u_{M} t_{2}^{2}-2 u_{M} t_{2} t_{3}+\frac{1}{2} u_{M} t_{3}^{2} \\
& +a_{0} t_{3}=v_{f}-v_{0} .
\end{aligned}
$$

The integration of the first scalar equation of (6) leads to $x_{1}(t)=\int_{0}^{t} x_{2}(\xi) d \xi$ and the interpolation of the final distance with $x_{1}\left(t_{3}\right)=s_{f}$ gives a cubic equation in the unknowns $t_{1}, t_{2}$, and $t_{3}$ :

$$
\begin{aligned}
\frac{1}{3} u_{M} t_{1}^{3} & -u_{M} t_{1}^{2} t_{3}+u_{M} t_{1} t_{3}^{2}-\frac{1}{3} u_{M} t_{2}^{3}+u_{M} t_{2}^{2} t_{3} \\
& -u_{M} t_{2} t_{3}^{2}+\frac{1}{6} u_{M} t_{3}^{3}+\frac{1}{2} a_{0} t_{3}^{2}+v_{0} t_{3}=s_{f} .
\end{aligned}
$$

Collecting equations (13), (15) and (16), the following system of nonlinear algebraic equations arises

$$
\left\{\begin{array}{l}
2 u_{M} t_{1}-2 u_{M} t_{2}+u_{M} t_{3}=a_{f}-a_{0} \\
-u_{M} t_{1}^{2}+2 u_{M} t_{1} t_{3}+u_{M} t_{2}^{2}-2 u_{M} t_{2} t_{3} \\
\quad+\frac{1}{2} u_{M} t_{3}^{2}+a_{0} t_{3}=v_{f}-v_{0} \\
\frac{1}{3} u_{M} t_{1}^{3}-u_{M} t_{1}^{2} t_{3}+u_{M} t_{1} t_{3}^{2}-\frac{1}{3} u_{M} t_{2}^{3}+u_{M} t_{2}^{2} t_{3} \\
\quad-u_{M} t_{2} t_{3}^{2}+\frac{1}{6} u_{M} t_{3}^{3}+\frac{1}{2} a_{0} t_{3}^{2}+v_{0} t_{3}=s_{f} .
\end{array}\right.
$$

Define the data of the minimum-time velocity planning as vector $\mathbf{p}:=\left(s_{f}, j_{M}, v_{0}, a_{0}, v_{f}, a_{f}\right)$ and considering the structure of the optimal jerk as described in Section 2, the solution set which is relevant for our problem can be introduced as follows

$$
\begin{aligned}
S_{\mathbf{p}}:= & \left\{\left(t_{1}, t_{2}, t_{3}\right) \in \mathbb{R}^{3}:\left(t_{1}, t_{2}, t_{3}\right) \quad\right. \text { satisfies (17) with } \\
& \left.0 \leq t_{1} \leq t_{2} \leq t_{3}, t_{3}>0, u_{M} \in\left\{-j_{M},+j_{M}\right\}\right\} .
\end{aligned}
$$

Remark 1: A triple $\left(t_{1}, t_{2}, t_{3}\right)$ contained in $S_{\mathbf{p}}$ is associated to $u_{M}=+j_{M}$ or $u_{M}=-j_{M}$. For simplicity, we denote it $\left(t_{1}, t_{2}, t_{3}\right)_{[+]}$or $\left(t_{1}, t_{2}, t_{3}\right)_{[-]}$if it is matched with the former or the latter respectively.

Proposition 2: Set $S_{\mathbf{p}}$ is not empty for any data set $\mathbf{p}$ and contains the solution to the MTVP problem (cf. (1)(5)) for which the sought minimum-time is given by

$$
t_{f}^{*}=\min _{\left(t_{1}, t_{2}, t_{3}\right) \in S_{\mathbf{p}}} t_{3} .
$$

Proof: For any data set $\mathbf{p}$, the MTVP problem is equivalent to a minimum-time input-constrained feedforward control problem for the triple integrator model (6) which is a controllable system. Hence, by Pontryagin's Maximum Principle, there exists a unique solution to the MTVP problem (cf. Theorem 1 and (10)) for which $u^{*}(t)$ has the structure described in Fig. 2 and (11). By integration of model (6) and by imposition of the boundary conditions (2)-(4), we have deduced that switching times $t_{1}, t_{2}$ and final time $t_{3}$ of the optimal solution must satisfy the algebraic system (17). Hence, $S_{\mathbf{p}}$ is not empty and contains the optimal solution. However, other non-optimal triples $\left(t_{1}, t_{2}, t_{3}\right)$ may be found in $S_{\mathbf{p}}$ cf. the examples of Section 5 - so that the sought optimal solution needs to be determined by (19), i.e., by minimizing the final time $t_{3}$ of all the triples in $S_{\mathbf{p}}$.

Proposition 2 shows that the original minimum-time velocity planning problem has been completely reduced to an algebraic problem. Indeed, the minimum-time $t_{f}^{*}$ will be determined by a finite search among the elements of the solution set $S_{\mathbf{p}}$. Construction of $S_{\mathbf{p}}$ can be performed by algebraic reduction of system (17). The first equation of (17) implies

$$
t_{1}=t_{2}-\frac{t_{3}}{2}+\frac{a_{f}-a_{0}}{2 u_{M}} .
$$


By substituting the expression (20) of $t_{1}$ into the second equation of system (17) we obtain

$$
t_{2}=\frac{\frac{3}{4} u_{M} t_{3}^{2}-\frac{1}{2}\left(3 a_{f}-a_{0}\right) t_{3}+\frac{1}{4 u_{M}}\left(a_{f}-a_{0}\right)^{2}+v_{f}-v_{0}}{u_{M} t_{3}-a_{f}+a_{0}},
$$

which is a valid expression for the switching time $t_{2}$ provided that

$$
u_{M} t_{3}-a_{f}+a_{0} \neq 0 .
$$

Eventually, by substitution of (20) and (21) into the third equation of system (17), a quartic equation in the unknown $t_{3}$ is obtained:

$$
\begin{aligned}
& \frac{1}{32} u_{M}^{2} t_{3}^{4}+\frac{1}{8} u_{M}\left(a_{0}-a_{f}\right) t_{3}^{3} \\
& +\left(\frac{1}{2} u_{M}\left(v_{0}+v_{f}\right)-\frac{1}{16}\left(a_{0}^{2}+a_{f}^{2}\right)-\frac{3}{8} a_{0} a_{f}\right) t_{3}^{2} \\
& +\left(\frac{1}{8} \frac{a_{0} a_{f}}{u_{M}}\left(a_{0}-a_{f}\right)-\frac{1}{24} \frac{a_{0}^{3}-a_{f}^{3}}{u_{M}}\right. \\
& \left.+a_{0} v_{f}-a_{f} v_{0}-u_{M} s_{f}\right) t_{3} \\
& \quad-\frac{1}{96} \frac{a_{0}^{4}+a_{f}^{4}}{u_{M}^{2}}+\frac{1}{24} \frac{a_{0} a_{f}}{u_{M}^{2}}\left(a_{0}^{2}+a_{f}^{2}\right)-\frac{1}{16} \frac{a_{0}^{2} a_{f}^{2}}{u_{M}^{2}} \\
& \quad-\frac{1}{2}\left(v_{0}^{2}+v_{f}^{2}\right)+v_{0} v_{f}-a_{0} s_{f}+a_{f} s_{f}=0 .
\end{aligned}
$$

This quartic equation can admit four real roots at most that generate four solutions of (17) at most using relations (20), (21). Considering that $u_{M}$ can be $j_{M}$ or $-j_{M}$ we may obtain at most eight triples $\left(t_{1}, t_{2}, t_{3}\right)$ contained in the solution set $S_{\mathbf{p}}$. This fact along with the property that the roots of any quartic equation can be found by finite elementary operations and root extractions (cf. e.g., [21]) has the remarkable consequence that the time-optimal velocity planning is solvable in a finite number of steps (cf. Section 4).

\subsection{The degenerate case}

The triples $\left(t_{1}, t_{2}, t_{3}\right)$ generated by the quartic equation (23) and expressions (20), (21) are only valid if condition (22) is satisfied. When this condition is not satisfied, $t_{3}$ takes the peculiar value

$$
t_{3}=\frac{a_{f}-a_{0}}{u_{M}} .
$$

Considering that $t_{3}$ must be strictly positive, i.e., $t_{3}>0$, it follows that

$$
a_{f} \neq a_{0} \text { and } u_{M}=\operatorname{sign}\left(a_{f}-a_{0}\right) j_{M},
$$

where sign denotes the standard sign function. If (24) holds, the first equation of system (17) becomes $2 u_{M} t_{1}$ $-2 u_{M} t_{2}=0$ which is equivalent to $t_{1}=t_{2}$. Therefore, system (17) with the imposition of (24) and (25) takes the form

$$
\left\{\begin{array}{l}
t_{1}=t_{2} \\
\frac{1}{2} \frac{\left|a_{f}-a_{0}\right|\left(a_{f}+a_{0}\right)}{j_{M}}=v_{f}-v_{0} \\
\frac{1}{6} \frac{\left(a_{f}-a_{0}\right)^{3}}{j_{M}^{2}}+\frac{1}{2} a_{0} \frac{\left(a_{f}-a_{0}\right)^{2}}{j_{M}^{2}}+v_{0} \frac{\left|a_{f}-a_{0}\right|}{j_{M}}=s_{f} .
\end{array}\right.
$$

The second and third equations of (26) do not depend on the unknowns $t_{1}$ and $t_{2}$. This shows that the case at hand is a degenerate one that arises only when the given interpolating data satisfy the particular relations:

$$
\left\{\begin{array}{l}
a_{f} \neq a_{0} \\
\frac{1}{2} \frac{\left|a_{f}-a_{0}\right|\left(a_{f}+a_{0}\right)}{j_{M}}=v_{f}-v_{0} \\
\frac{1}{6} \frac{\left(a_{f}-a_{0}\right)^{3}}{j_{M}^{2}}+\frac{1}{2} a_{0} \frac{\left(a_{f}-a_{0}\right)^{2}}{j_{M}^{2}}+v_{0} \frac{\left|a_{f}-a_{0}\right|}{j_{M}}=s_{f} .
\end{array}\right.
$$

When relations (27) hold, the solution set $S_{\mathbf{p}}$ contains a subset of degenerate solutions according to

$$
\left\{\left(\xi, \xi, \frac{\left|a_{f}-a_{0}\right|}{j_{M}}\right): \xi \in\left[0, \frac{\left|a_{f}-a_{0}\right|}{j_{M}}\right]\right\} \subseteq S_{\mathbf{p}} .
$$

From (11) it follows that all the triples $\left(\xi, \xi, \frac{\left|a_{f}-a_{0}\right|}{j_{M}}\right)$ contained in $S_{\mathbf{p}}$ actually correspond to just one jerk profile defined as:

$$
u(t)=\operatorname{sign}\left(a_{f}-a_{0}\right) j_{M}, t \in\left[0, t_{3}\right] ; t_{3}=\frac{\left|a_{f}-a_{0}\right|}{j_{M}} .
$$

Even when the degeneracy conditions (27) are satisfied, the solution set $S_{\mathbf{p}}$ may contain "standard" triples $\left(t_{1}, t_{2}\right.$, $t_{3}$ ), i.e., triples for which $t_{3} \neq \frac{\left|a_{f}-a_{0}\right|}{j_{M}}$ (cf. Example 4 in Section 5). However, these standard triples do not need to be determined according to this result.

Proposition 3: Suppose that the degeneracy conditions (27) hold. Then the time-optimal jerk profile is given by (29), i.e.,

$$
t_{f}^{*}=\frac{\left|a_{f}-a_{0}\right|}{j_{M}}, u^{*}(t)=\operatorname{sign}\left(a_{f}-a_{0}\right) j_{M}, t \in\left[0, t_{f}^{*}\right] .
$$

Proof: The degeneracy conditions (27) ensure the existence of the degenerate triples (28) all corresponding to the jerk constant profile $u(t)$ defined in (29). This jerk is actually the time-optimal (i.e., minimum-time) $u^{*}(t)$ reported in (30). To prove this, consider a standard triple $\left(t_{1}, t_{2}, t_{3}\right)$ of $S_{\mathbf{p}}$. It satisfies the inequality

$$
t_{3} \neq \frac{\left|a_{f}-a_{0}\right|}{j_{M}} \text {. }
$$

We will show that necessarily

$$
t_{3}>\frac{\left|a_{f}-a_{0}\right|}{j_{M}} \text {. }
$$


From the first equation of (17), the final time $t_{3}$ can be expressed as

$$
t_{3}=\frac{a_{f}-a_{0}}{u_{M}}+2\left(t_{2}-t_{1}\right) .
$$

Both the values $+j_{M}$ and $-j_{M}$ can be assumed by $u_{M}$, so that we distinctly consider the cases

$$
u_{M}=\operatorname{sign}\left(a_{f}-a_{0}\right) j_{M}
$$

and

$$
u_{M}=-\operatorname{sign}\left(a_{f}-a_{0}\right) j_{M} .
$$

From the case (34), (33) becomes

$$
t_{3}=\frac{\left|a_{f}-a_{0}\right|}{j_{M}}+2\left(t_{2}-t_{1}\right)
$$

and taking into account both relation $t_{2} \geq t_{1}$ (cf. the definition of $S_{\mathbf{p}}$ in (18)) and (31), we deduce (32).

The case given by (35) implies

$$
t_{3}=-\frac{\left|a_{f}-a_{0}\right|}{j_{M}}+2\left(t_{2}-t_{1}\right)
$$

and

$$
t_{2}-t_{1}=\left(t_{3}-t_{2}\right)+t_{1}+\frac{\left|a_{f}-a_{0}\right|}{j_{M}} .
$$

Considering the triple ordering $0 \leq t_{1} \leq t_{2} \leq t_{3}$ the following relations hold:

$$
t_{2}-t_{1} \geq \frac{\left|a_{f}-a_{0}\right|}{j_{M}}, t_{2} \geq \frac{\left|a_{f}-a_{0}\right|}{j_{M}}, t_{3} \geq \frac{\left|a_{f}-a_{0}\right|}{j_{M}} .
$$

The last inequality of (37) with (31) proves (32) again. This concludes the proof by virtue of Proposition 2 .

Remark 2: The degenerate case in the MTVP problem only emerges when the initial and final conditions, i.e., $v_{0}, a_{0}$ and $v_{f}, a_{f}, s_{f}$, satisfy the special expressions given in (27). An interpretation of this degeneracy follows. Given the initial velocity and acceleration $v_{0}, a_{0}$, the final conditions $v_{f}, a_{f}, s_{f}$ are the unique values corresponding to an extreme, constant jerk $u^{*}(t)=j_{M}$ or $-j_{M}$ applied for a given time interval $\left[0, t_{3}\right]$. Indeed, $a_{f}$ is dictated by equation (24) and $v_{f}, s_{f}$ by the second and third expression in (27) respectively.

\subsection{The case of initial or final nil conditions}

A special case of the MTVP problem (1)-(5) emerges when $v_{0}=0, a_{0}=0$ (initial nil conditions) or $v_{f}=0, a_{f}=0$ (final nil conditions). As shown by Corollary 1 in this case if a jerk profile $u(t)$ defined in (11) satisfies the boundary conditions, then it is the unique time-optimal $u^{*}(t)$. This fact has a consequence on the solution set's cardinality as the following result presents.

Proposition 4: Suppose that $v_{0}=0, a_{0}=0$ or $v_{f}=0$, $a_{f}=0$ and the degeneracy conditions (27) do not hold.
Then the solution set $S_{\mathbf{p}}$ has cardinality equal to 1 or equal to 2 . In the latter case, both elements of $S_{\mathrm{p}}$ still correspond to a unique time-optimal $u^{*}(t)$ that has just one switching time.

Proof: The solution set $S_{\mathbf{p}}$ (cf. (18)) contains all the triples $\left(t_{1}, t_{2}, t_{3}\right)$ that define a jerk profile $u(t)$ (cf. (11)) satisfying the boundary conditions of the MTVP problem. If $v_{0}=0, a_{0}=0$ or $v_{f}=0, a_{f}=0$ then Corollary 1 says that $u(t)$ is actually the unique time-optimal $u^{*}(t)$.

On the assumption that the degeneracy conditions (27) do not hold, by Proposition 3, $u^{*}(t)$ must have one switching time at least. Consider the case for which $u^{*}(t)$ has two distinct switching times (i.e., $0<t_{1}<t_{2}<t_{3}$ ). Then $S_{\mathbf{p}}$ can only contain the sole triple $\left(t_{1}, t_{2}, t_{3}\right)$ : the cardinality of $S_{\mathbf{p}}$ is 1 .

On the other hand, assume that $u^{*}(t)$ has just one switching time denoted by $s_{t}$ and the final minimum-time is $t_{f}^{*}$. If $u^{*}(t)$ has positive initial jerk then both distinct triples $\left(s_{t}, t_{f}^{*}, t_{f}^{*}\right)_{[+]}$and $\left(0, s_{t}, t_{f}^{*}\right)_{[-]}$belong to $S_{\mathbf{p}}$ and correspond to the same $u^{*}(t)$. No other triples can correspond to $u^{*}(t)$ : hence, the cardinality of $S_{\mathbf{p}}$ is 2 . Analogously, when $u^{*}(t)$ has negative initial jerk, the sole triples in $S_{\mathbf{p}}$ that correspond to $u^{*}(t)$ are the distinct $\left(s_{t}, t_{f}^{*}, t_{f}^{*}\right)_{[-]}$and $\left(0, s_{t}, t_{f}^{*}\right)_{[+]}$. Cardinality of $S_{\mathbf{p}}$ is confirmed to be 2 .

\section{THE MTVP ALGORITHM}

In this section, the Minimum-Time Velocity Planning (MTVP) algorithm will be presented by exploiting the algebraic approach exposed in Section 3. Hence, using pseudocode, the MTVP algorithm can be composed as follows. It uses the Quartic Equation (QE) procedure immediately defined after the main algorithm.

\section{MTVP Algorithm}

Input: $v_{0}, a_{0}, v_{f}, a_{f}, s_{f} \neq 0, j_{M}>0$.

Output: $t_{1}, t_{2}, t_{f}^{*}>0$, and $u_{M}$ of the time-optimal jerk $u^{*}(t)$

begin

if conditions (27) are satisfied then

$$
\begin{aligned}
& \quad\left(t_{1}, t_{2}, t_{f}^{*}\right):=\left(0,0, \frac{\left|a_{f}-a_{0}\right|}{j_{M}}\right) ; \\
& u_{M}:=\operatorname{sign}\left(a_{f}-a_{0}\right) j_{M} ; \\
& \text { exit } \\
& \text { endif } \\
& u_{M}:=j_{M} ; \alpha:=0 ; \\
& \text { QE Procedure; } \\
& \text { if } \alpha=0 \text { then } \\
& \quad u_{M}:=-j_{M} ; \\
& \quad \text { QE } \operatorname{Procedure;} \\
& \quad\left(t_{1}, t_{2}, t_{f}^{*}\right):=\left(\beta_{1}, \beta_{2}, \beta_{3}\right) ; \\
& \quad \text { exit } \\
& \text { endif } \\
& \left(t_{1}, t_{2}, t_{f}^{*}\right):=\left(\beta_{1}, \beta_{2}, \beta_{3}\right) ; \\
& \text { if } \quad\left(v_{0}=0 \wedge a_{0}=0\right) \vee\left(v_{f}=0 \wedge a_{f}=0\right) \text { then }
\end{aligned}
$$


exit

endif

$$
\begin{aligned}
& u_{M}:=-j_{M} ; \alpha:=0 ; \\
& \text { QE Procedure; } \\
& \text { if } \alpha=0 \text { then } \\
& u_{M}:=j_{M} ; \\
& \text { exit } \\
& \text { endif } \\
& \text { if } \beta_{3}<t_{f}^{*} \text { then } \\
& \qquad\left(t_{1}, t_{2}, t_{f}^{*}\right):=\left(\beta_{1}, \beta_{2}, \beta_{3}\right) ; \\
& \text { else } \\
& u_{M}:=j_{M} ; \\
& \text { endif }
\end{aligned}
$$$$
\text { end }
$$

\section{QE Procedure}

\section{begin}

Compute the positive real roots of equation (23), store them in vector $\mathbf{r}:=\left(t_{31}, t_{32}, \ldots, t_{3 l}\right),(l \leq 4)$ satisfying $t_{31}<t_{32}<\ldots<t_{3 l}$ and

$$
\begin{aligned}
& u_{M} t_{3 i}-a_{f}+a_{0} \neq 0, i=1,2, \ldots, l \\
& \text { if } \mathbf{r} \text { is empty then } \\
& \text { return } \\
& \text { endif }
\end{aligned}
$$

$$
\begin{aligned}
& \text { for } i=1, \ldots, l \text { do } \\
& t_{2 i}:=\frac{\frac{3}{4} u_{M} t_{3 i}^{2}-\frac{1}{2}\left(3 a_{f}-a_{0}\right) t_{3 i}+\frac{1}{4 u_{M}}\left(a_{f}-a_{0}\right)^{2}+v_{f}-v_{0}}{u_{M} t_{3 i}-a_{f}+a_{0}} ; \\
& \text { if } 0 \leq t_{2 i} \leq t_{3 i} \text { then } \\
& t_{1 i}:=t_{2 i}-\frac{1}{2} t_{3 i}^{2}+\frac{1}{2} \frac{a_{f}-a_{0}}{u_{M}} ; \\
& \text { if } 0 \leq t_{1 i} \leq t_{2 i} \text { then } \\
& \alpha:=1 \text {; } \\
& \left(\beta_{1}, \beta_{2}, \beta_{3}\right)=\left(t_{1 i}, t_{2 i}, t_{3 i}\right) \text {; } \\
& \text { return } \\
& \text { endif } \\
& \text { endif } \\
& \text { endfor } \\
& \text { return }
\end{aligned}
$$$$
\text { end }
$$

Remark 3: In the MTVP algorithm, note the roles of the Degeneracy Test and of the Nil Conditions Test. The former test is based on Proposition 3 and if it is satisfied the time-optimal jerk can be immediately provided without the need to solve the quartic equation. If the Nil Conditions Test is satisfied then Proposition 4 permits to exit the algorithm as soon as a bang-bang jerk profile satisfying the boundary conditions is found.

\section{EXAMPLES}

Example 1: Consider a MTVP problem where the distance to be traveled is $s_{f}=3.25 \mathrm{~m}$, the allowed maximum jerk is $j_{M}=0.5 \mathrm{~m} / \mathrm{s}^{3}$, the initial velocity and acceleration are $v_{0}=0 \mathrm{~m} / \mathrm{s}$ and $a_{0}=0 \mathrm{~m} / \mathrm{s}^{2}$, and the final velocity and acceleration are $v_{f}=2.25 \mathrm{~m} / \mathrm{s}$ and $a_{f}=1.5 \mathrm{~m} / \mathrm{s}^{2}$. This data corresponds to parameter vector $\mathbf{p}=(3.25,0.5,0,0,2.25,1.5)$ and the associated solution set is

$$
S_{\mathbf{p}}=\left\{(1,3,7)_{[+]}\right\} .
$$

Hence, the time-optimal jerk, that is associated to triple $(1,3,7)_{[+]}$, has initial jerk value $u_{M}=+0.5 \mathrm{~m} / \mathrm{s}^{3}$, switching times $t_{1}=1 \mathrm{~s}, t_{2}=3 \mathrm{~s}$, and final minimumtime $t_{f}^{*}=7 \mathrm{~s}$ (cf. (11) and Remark 1). The corresponding profiles of jerk, acceleration, velocity and distance are depicted in Figs. 3 and 4.

Note that the case at hand is a non-degenerate one because the degeneracy conditions (27) are not satisfied and $S_{\mathbf{p}}$ has cardinality one according to Proposition 4 (the problem data corresponds to initial nil conditions, i.e., $v_{0}=0$ and $\left.a_{0}=0\right)$. The time-optimal triple $(1,3,7)_{[+]}$in $S_{\mathbf{p}}$ can be readily determined by setting $u_{M}=+0.5$ and computing the positive real roots of the quartic equation (23):

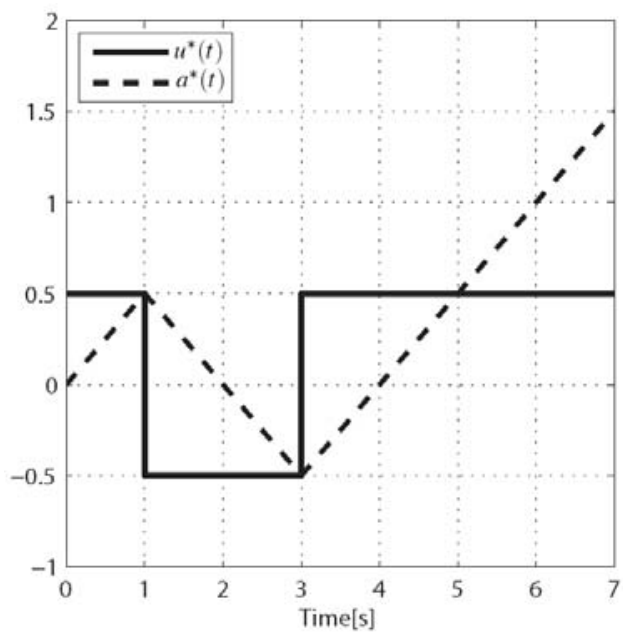

Fig. 3. The optimal profiles of jerk $u^{*}(t)$ and acceleration $a^{*}(t)$ for Example 1.

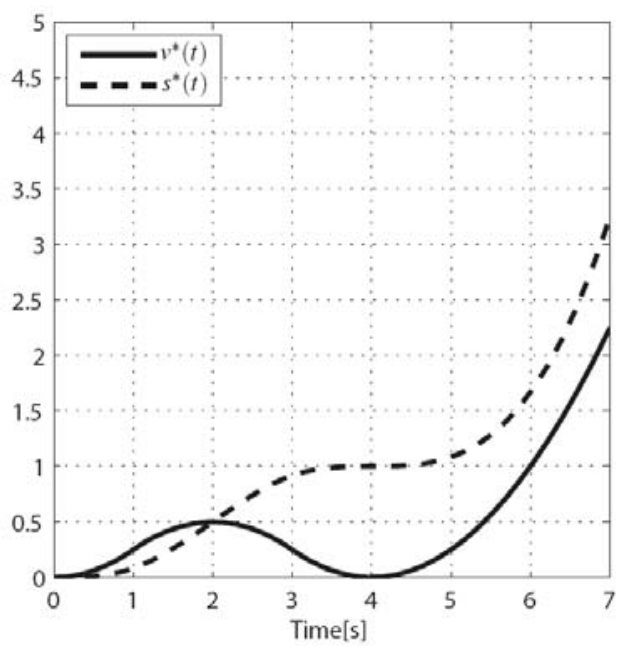

Fig. 4. The optimal profiles of velocity $v^{*}(t)$ and distance $s^{*}(t)$ for Example 1. 


$$
\frac{1}{128} t_{3}{ }^{4}-\frac{3}{32} t_{3}{ }^{3}+\frac{27}{64} t_{3}{ }^{2}-\frac{43}{32} t_{3}+\frac{273}{128}=0
$$

they are $t_{31}=3$ and $t_{32}=7$. The first root, i.e., $t_{31}=3$, has to be discarded because it violates condition (22) whereas the second one is acceptable. Hence, by applying expressions (20), (21) with $t_{32}=7$, triple $(1,3,7)_{[+]}$is obtained (cf. in Section 4, the QE procedure and the MTVP algorithm).

Example 2: Let be the case of $s_{f}=13 \mathrm{~m}, j_{M}=3$ $\mathrm{m} / \mathrm{s}^{3}, \quad v_{0}=0 \mathrm{~m} / \mathrm{s}, \quad a_{0}=1 \mathrm{~m} / \mathrm{s}^{2}, \quad v_{f}=1 \mathrm{~m} / \mathrm{s}$ and $a_{f}=-5$ $\mathrm{m} / \mathrm{s}^{2}$. The solution set associated to this MTVP problem is

$$
S_{p}=\left\{(1,4,4)_{[+]},\left(\frac{2}{3}, \frac{7}{3}, \frac{16}{3}\right)_{[-]},(0,1,4)_{[-]}\right\} .
$$

The time-optimal jerk is given by triple $(1,4,4)_{[+]}$: $u_{M}=+3 \mathrm{~m} / \mathrm{s}^{3}, t_{1}=1 \mathrm{~s}, t_{2}=t_{3}=t_{f}^{*}=4 \mathrm{~s}$. Note that there is actually just one switching time and the same optimal jerk profile is also given by the equivalent triple $(0,1,4)_{[-]}$(cf. (11) and Remark 1). The other third triple

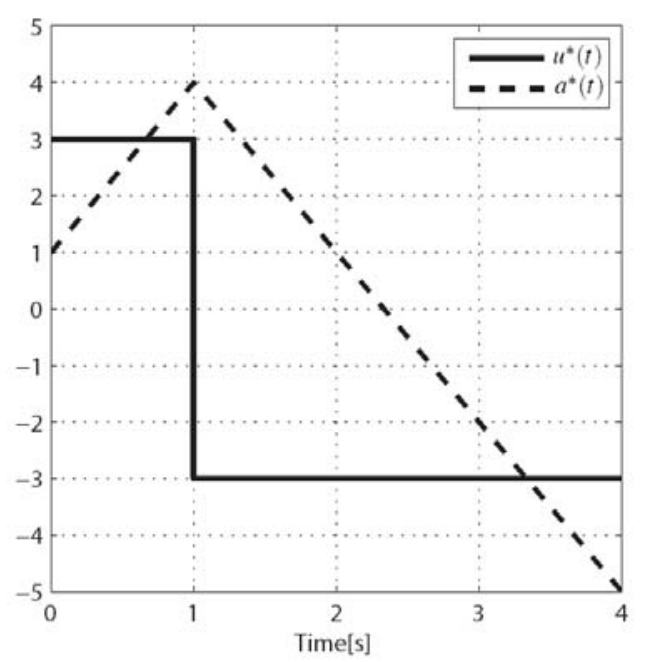

Fig. 5. The optimal profiles of jerk $u^{*}(t)$ and acceleration $a^{*}(t)$ for Example 2.

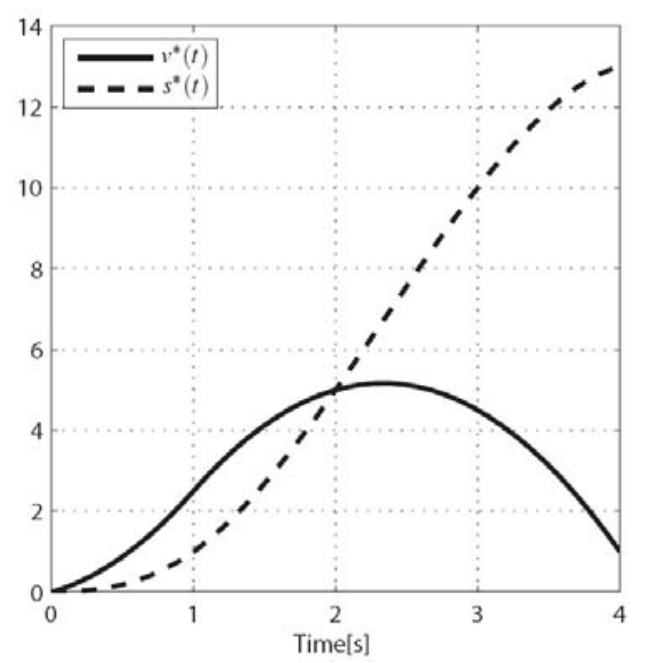

Fig. 6. The optimal profiles of velocity $v^{*}(t)$ and distance $s^{*}(t)$ for Example 2 . in $S_{\mathbf{p}}$ gives a jerk profile leading to a velocity planning that satisfies all the conditions (2)-(5), but evidently it is not a minimum-time planning. The optimal profiles for this case are reported in Figs. 5 and 6.

Example 3: Let be given the following data: $s_{f}=20 \mathrm{~m}, \quad j_{M}=0.75 \mathrm{~m} / \mathrm{s}^{3}, \quad v_{0}=5 \mathrm{~m} / \mathrm{s}, \quad a_{0}=1 \mathrm{~m} / \mathrm{s}^{2}$, $v_{f}=10 \mathrm{~m} / \mathrm{s}$ and $a_{f}=2 \mathrm{~m} / \mathrm{s}^{2}$. This minimum-time planning problem has the following solution set (triples are expressed up to centiseconds):

$$
\begin{aligned}
S_{\mathbf{p}}=\{ & (1.83,2.55,2.76)_{[+]},(0.11,2.18,2.80)_{[-]}, \\
& \left.(4.99,13.43,15.53)_{[-]}\right\} .
\end{aligned}
$$

Triple $(1.83,2.55,2.76)_{[+]}$defines the time-optimal jerk with minimum-time $t_{f}^{*}=2.76 \mathrm{~s}$, switching times $t_{1}=$ $1.83 \mathrm{~s}, t_{2}=2.55 \mathrm{~s}$ and initial jerk $u_{M}=+0.75 \mathrm{~m} / \mathrm{s}^{3}$. The corresponding profiles of jerk, acceleration, velocity and distance are depicted in Figs. 7 and 8.

Example 4: Consider the case of $s_{f}=8.25 \mathrm{~m}$, $j_{M}=0.5 \mathrm{~m} / \mathrm{s}^{3}, \quad v_{0}=2 \mathrm{~m} / \mathrm{s}, \quad a_{0}=1 \mathrm{~m} / \mathrm{s}^{2}, \quad v_{f}=2.75 \mathrm{~m} / \mathrm{s}$,

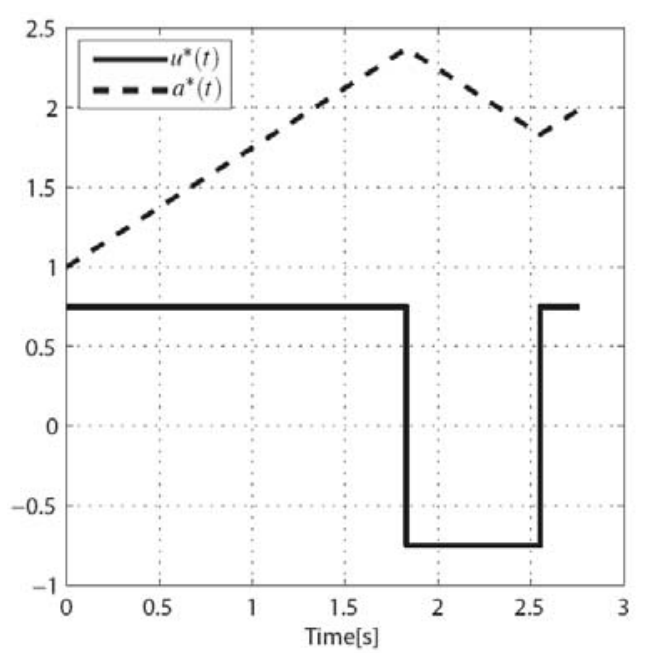

Fig. 7. The optimal profiles of jerk $u^{*}(t)$ and acceleration $a^{*}(t)$ for Example 3.

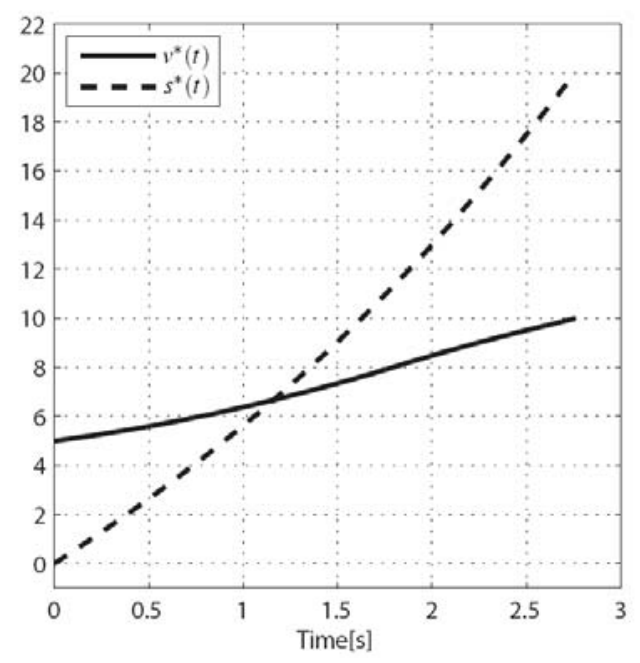

Fig. 8. The optimal profiles of velocity $v^{*}(t)$ and distance $s^{*}(t)$ for Example 3 . 
and $a_{f}=-0.5 \mathrm{~m} / \mathrm{s}^{2}$. In this instance, differently from all the previous examples, the degeneracy test of the MTVP problem is positive, i.e., conditions (27) are satisfied. By virtue of Proposition 3, the time-optimal jerk, that has constant value over its time interval, is given by

$$
t_{f}^{*}=3 \mathrm{~s}, \quad u^{*}(t)=-0.5 \mathrm{~m} / \mathrm{s}^{3}, \quad t \in\left[0, t_{f}^{*}\right] .
$$

The solution set is (cf. (28))

$$
\begin{aligned}
& S_{p}=\left\{(0,3,3)_{[+]},(\xi, \xi, 3)_{[-]} \text {with } \xi \in[0,3],\right. \\
&\left.(5.46,12.39,16.86)_{[-]}\right\} .
\end{aligned}
$$

Note that triples $(0,3,3)_{[+]}$and $(\xi, \xi, 3)_{[-]}$with $\xi \in$ $[0,3]$ all correspond to the same optimal degenerate jerk defined above in (38). Also note the presence in $S_{\mathbf{p}}$ of the standard triple $(5.46,12.39,16.86)_{[-]}$whose final time $t_{3}=16.86 \mathrm{~s}$ is however much greater than the minimumtime $t_{f}^{*}=3 \mathrm{~s}$. The optimal profiles of this velocity planning are reported in Figs. 9 and 10.

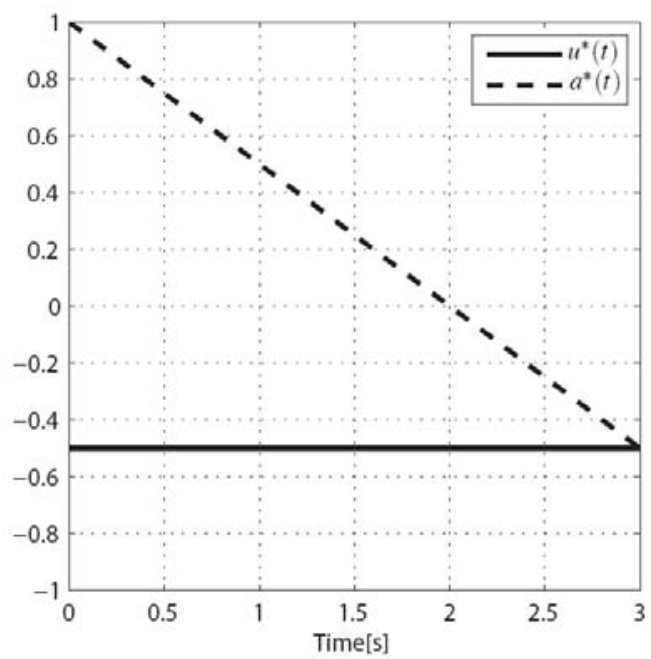

Fig. 9. The optimal profiles of jerk $u^{*}(t)$ and acceleration $a^{*}(t)$ for Example 4 .

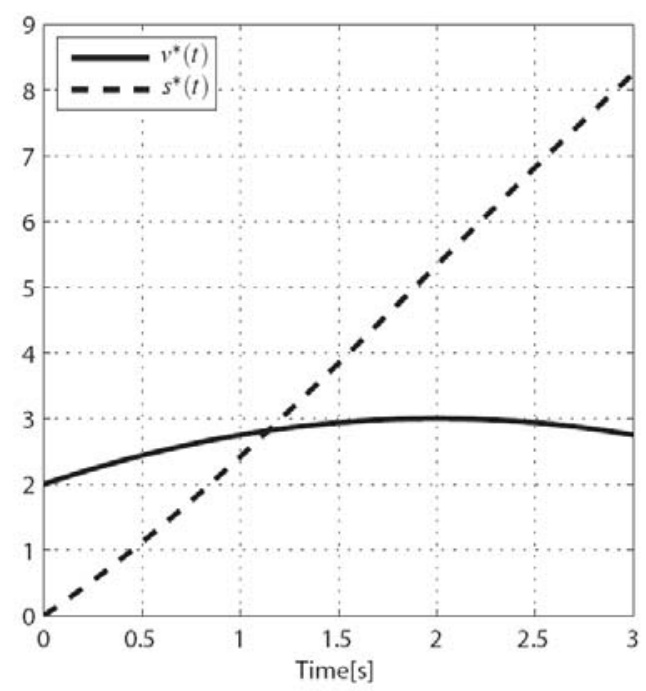

Fig. 10. The optimal profiles of velocity $v^{*}(t)$ and distance $s^{*}(t)$ for Example 4.

\section{CONCLUSION}

The paper has proposed a velocity planning scheme that achieves a true global minimum-time along with smooth motion - acceleration is continuous, jerk is bounded and arbitrary boundary conditions. This planning can be easily executed in real-time because the devised MTVP algorithm relies on the solution of an algebraic quartic equation whose roots can be straightforwardly computed by well-known closed-form expressions [21]. Application details on using a velocity planning to permit a wheeled mobile robot to follow a given path with the prescribed velocity profile are given, for example, in [5,22].

A possible drawback of this velocity planning is the lack of explicit constraints to be added in the MTVP problem (1)-(5) on velocities and accelerations during the planned time interval. These constraints, which may be very important from an application standpoint due to the inevitable limitations of physical actuators, can be viewed as state constraints on the triple integrator system (6). In such a constrained case, the time-optimal velocity planning cannot then lead to the standard bang-bang control but to a form of generalized bang-bang control [23]. An approximate solution to the constrained MTVP problem (i.e., the MTVP problem with velocity and acceleration constraints during the planned time interval) has been proposed in [24]. This solution is useful for offline planning but it is difficult to implement in real-time applications because it relies on linear programming. Perhaps, future research on the constrained MTVP problem should consider the possibility to study and exploit the structure of the associated time-optimal jerk in order to device a fast solution algorithm.

\section{REFERENCES}

[1] K. G. Shin and N. D. McKay, "Minimum-time control of robotic manipulators with geometric path constraints," IEEE Trans. on Automatic Control, vol. AC-30, no. 6, pp. 531-541, 1985.

[2] L. E. Dubins, "On curves of minimal length with a constraint on average curvature and with prescribed initial and terminal positions and tangents," American Journal of Mathematics, vol. 79, no. 79, pp. 497-517, 1957.

[3] J. A. Reeds and R. A. Shepp, "Optimal paths for a car that goes both forward and backward," Pacific Journal of Mathematics, vol. 145, no. 2, pp. 367393, 1990.

[4] K. Kant and S.W. Zucker, "Toward efficient trajectory planning: the path-velocity decomposition," Int. J. of Robotics Research, vol. 5, no. 3, pp. 72-89, 1986.

[5] C. Guarino Lo Bianco, A. Piazzi, and M. Romano, "Smooth motion generation for unicycle mobile robots via dynamic path inversion," IEEE Trans. on Robotics, vol. 20, no. 5, pp. 884-891, October 2004.

[6] P. Lucibello and G. Oriolo, "Robust stabilization via iterative state steering with an application to chained-form systems," Automatica, vol. 37, no. 1, pp. 71-79, January 2001. 
[7] A. Piazzi, C. Guarino Lo Bianco, M. Bertozzi, A. Fascioli, and A. Broggi, "Quintic $\mathrm{G}^{2}$-splines for the iterative steering of vision-based autonomous vehicles," IEEE Trans. on Intelligent Transportations Systems, vol. 3, no. 1, pp. 27-36, March 2002.

[8] K. C. Koh, H. S. Aum, and H. S. Cho, "A minimum-time motion planning method based on phase space analysis," Proc. of the IEEE International Conference on Control Applications, pp. 273-278, Hawaii, USA, August 1999.

[9] M. Prado, A. Simon, E. Carabias, A. Perez, and F. Ezquerro, "Optimal velocity planning of wheeled mobile robots on specific paths in static and dynamic environments," Journal of Robotic Systems, vol. 20, no. 12, pp. 737-754, 2003.

[10] C. Guarino Lo Bianco, A. Piazzi, and M. Romano, "Velocity planning for autonomous vehicles," Proc. of the IEEE Intelligent Vehicles Symposium, pp. 413-418, Parma, Italy, June 2004.

[11] C. Guarino Lo Bianco and M. Romano, "Bounded Velocity Planning for Autonomous Vehicles," Proc. of the IEEE/RSJ Int. Conf. on Intelligent Robots and Systems, pp. 4068-4073, Edmonton, Canada, August 2005.

[12] C. Guarino Lo Bianco and M. Romano, "Optimal velocity planning for autonomous vehicles considering curvature constraints," Proc. of the IEEE International Conference on Robotics and Automation, pp. 2706-2711, Roma, Italy, April 2007.

[13] R. Solea and U. Nunes, "Trajectory planning with velocity planner for fully-automated passenger vehicles," Proc. of the IEEE Intelligent Transportation Systems Conference, pp. 474-480, Toronto, Canada, September 2006.

[14] J. S. Gyorfi and C.-H. Wu, "A minimum-jerk speed-planning algorithm for coordinated planning and control of automated assembly manufacturing," IEEE Trans. on Automation Science and Engineering, vol. 3, no. 4, pp. 454-462, October 2006.

[15] L. Biagiotti and C. Melchiorri, Trajectory Planning for Automatic Machines and Robots, SpringerVerlag, Berlin, Germany, 2008.

[16] S. M. LaValle, Planning Algorithms, Cambridge University Press, 2006.

[17] G. Lini and A. Piazzi, "Minimum-time velocity planning with arbitrary boundary conditions," in K. R. Kozlowski (editor) Robot Motion and Control, pp. 287-296, Springer, Czerniejewo, Poland, June 2009.

[18] L. S. Pontryagin, V. G. Boltyanskii, R. V. Gamkrelidze, and E. F. Mishchenco, The Mathematical Theory of Optimal Processes, Wiley, New York, USA, 1962.

[19] E. D. Sontag, Mathematical Control Theory: Deterministic Finite Dimensional Systems, 2nd edition, Springer-Verlag, New York, 1998.

[20] A. A. Agrachev and Y. L. Sachkov, Control Theory from the Geometric Viewpoint, Springer-Verlag, Berlin, Germany, 2003.

[21] N. Jacobson, Basic Algebra I, 2nd edition, Dover
Publications, 2009.

[22] C. Guarino Lo Bianco, A. Piazzi, and M. Romano, "Smooth control of a wheeled omnidirectional robot," Proc. of the IFAC Symp. on Intelligent Autonomous Vehicles, Lisbon, Portugal, July 2004.

[23] L. Consolini and A. Piazzi, "Generalized bang-bang control for feedforward constrained regulation," Automatica, vol. 45, no. 10, pp. 2234-2243, 2009.

[24] G. Lini, L. Consolini, and A. Piazzi, "Minimumtime constrained velocity planning," Proc. of the 17th Mediterranean Conf. on Control and Automation, pp. 748-753, Thessaloniki, Greece, June 2009.

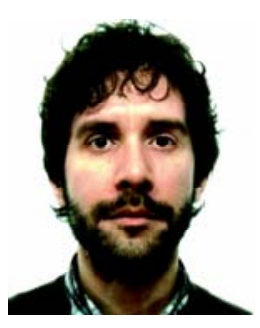

Gabriele Lini received his Master degree in Computer Engineering in 2008 and his Ph.D. degree in Information Technology in 2012 from the Department of Information Engineering both at the University of Parma, Italy. Currently, he is a postdoctoral researcher for the Functional Analysis Sector of the International School for Advanced Studies (SISSA) of Trieste, Italy, under the supervision of Prof. Claudio Altafini, and temporary researcher and software developer for the Glance Vision Technologies Srl of Trieste. His main research topics are in time-optimal control, feedforward/feedback control strategies, path planning, trajectory tracking, path following, consensus theory and artificial vision.

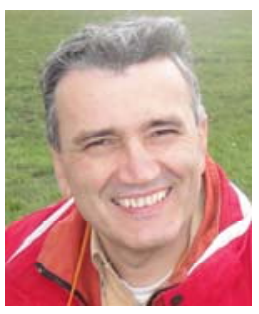

Aurelio Piazzi received his Laurea degree, magna cum laude, in Nuclear Engineering in 1982 and his Ph.D. degree in System Engineering in 1987, both from the University of Bologna, Italy. Since 1992 he has been affiliated with the University of Parma, Italy where he is a full professor of Systems and Control Engineering. His main research interests are in control theory, autonomous robotics, and mechatronics systems. His recent research activities have focused on inversion based and optimal feedforward methods, feedforward/feedback control strategies, and autonomous navigation and parking of wheeled robots and vehicles. He is a member of IEEE and SIAM. His research findings has been published over 100 scientific papers in international journals and conference proceedings.

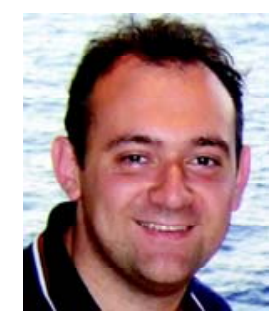

Luca Consolini is an assistant professor at the Department of Information Engineering, University of Parma, Italy from November 2010. He was born in Parma in 1976. In 2000 he obtained the laurea cum laude in Electronic Engineering at the University of Parma. In 2005, he received his Ph.D. at the University of Parma under the supervision of Prof. Aurelio Piazzi. In 2001 and 2002, he has been visiting scholar at the University of Toronto, Canada, under the supervision of Prof. Manfredi Maggiore. Since 2000, he collaborates actively with Prof. Mario Tosques, professor of mathematical analysis at the University of Parma. His main research topics are dynamic inversion for nonlinear systems, tracking and path following, formation control and time-optimal control. From 2005 to 2009 he has been a postdoc at the University of Parma. 